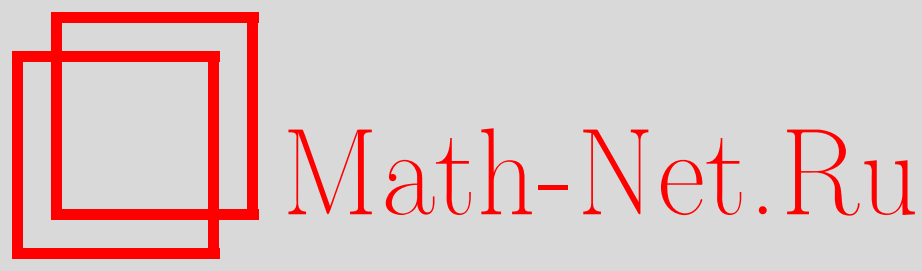

Е. Юрчишинова, М. Юрчишин, Р. Ремецки, П. Залом, Нарушение пространственной четности и турбулентное магнитное число Прандтля, ТМФ, 2013, том 176, номер 1, 150-159

DOI: https://doi.org/10.4213/tmf8485

Использование Общероссийского математического портала Math-Net.Ru подразумевает, что вы прочитали и согласны с пользовательским соглашением http://www.mathnet.ru/rus/agreement

Параметры загрузки:

IP : 52.87 .193 .239

26 апреля 2023 г., 13:11:41

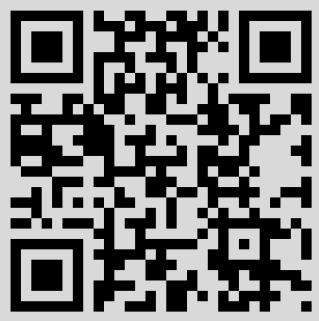




\title{
НАРУШЕНИЕ ПРОСТРАНСТВЕННОЙ ЧЕТНОСТИ И ТУРБУЛЕНТНОЕ МАГНИТНОЕ ЧИСЛО ПРАНДТЛЯ
}

\begin{abstract}
С использованием теоретико-полевых ренормгрупповых методов в двухпетлевом приближении исследуется влияние спиральности (нарушения пространственной четности) на турбулентное магнитное число Прандтля в модели кинематической магнитогидродинамической турбулентности, когда магнитное поле ведет себя как пассивная векторная величина, переносимая средой со спиральной турбулентностью, задаваемой стохастическим уравнением Навье-Стокса. Показано, что наличие спиральности уменьшает значение турбулентного магнитного числа Прандтля и что двухпетлевой спиральный вклад в турбулентное магнитное число Прандтля достигает $4.2 \%$ от его значения в отсутствие спиральности. Этот результат демонстрирует сильную устойчивость свойств диффузионных процессов в магнитном поле в турбулентных средах с нарушением пространственной четности по сравнению с соответствующими системами без спиральности.
\end{abstract}

Ключевые слова: развитая турбулентность, пассивный перенос, спиральность, ренормализационная группа.

DOI: $10.4213 / \operatorname{tmf} 8485$

\section{1. ВВЕДЕНИЕ}

Одной из характеристик диффузионных процессов в магнитном поле в проводящей среде является безразмерная величина отношения коэффициента кинематической вязкости к коэффициенту магнитной диффузии (сопротивления) - так называемое магнитное число Прандтля. Если проводящая среда находится в состоянии полностью развитой турбулентности, т.е. в состоянии магнитогидродинамической (МГД) турбулентности, то диффузионные процессы быстро ускоряются и описываются эффективным значением коэффициента магнитной диффузии - так называемой турбулентной магнитной диффузией (сопротивлением). Отношение турбулентной вязкости к турбулентной магнитной диффузии обычно называется турбулентным магнитным числом Прандтля $\mathrm{Pr}_{\mathrm{m}, \mathrm{t}}[1]$, [2] по аналогии с турбулентным числом

${ }^{*}$ Institute of Experimental Physics, Slovak Academy of Sciences, Košice, Slovakia. E-mail: zalom@saske.sk

† Объединенный институт ядерных исследований, Дубна, Московская обл., Россия 
Прандтля для тепловой диффузии [3], [4], которое принимает универсальное значение в пределе полностью развитой турбулентности.

С другой стороны, хорошо известно, что эффективным и действенным методом исследования универсальных свойств процессов в полностью развитой турбулентности является метод ренормализационной группы (РГ) [5], [6]. Недавно метод РГ был использован для вычисления двухпетлевого значения турбулентного магнитного числа Прандтля в рамках кинематической МГД-турбулентности в полностью симметричной изотропной турбулентной среде, описываемой стохастическим уравнением Навье-Стокса [7]. В работе [7] было показано, что двухпетлевая поправка к турбулентному магнитному числу Прандтля очень мала (меньше $2 \%$ от однопетлевого значения) и в то же время двухпетлевое значение турбулентного магнитного числа Прандтля, полученное в рамках кинематической МГД-турбулентности, равно двухпетлевому значению турбулентного числа Прандтля, вычисленному в соответствующей модели пассивно увлекаемого скалярного поля [8], [9], что также находится в хорошем согласии с экспериментальными значениями [3], [4].

Хотя согласно описанным выше двухпетлевым вычислениям представляется, что турбулентное магнитное число Прандтля устойчиво относительно пертурбативного разложения, тем не менее остаются открытыми ряд интересных вопросов. Например, вопрос о влиянии различных нарушений симметрии на процессы турбулентности глубоко внутри так называемого инерциального интервала $l \ll r \ll L$, т. е. вдали от внешнего (интегрального) масштаба $L$, где энергия вводится в систему для поддержания стационарного состояния, и вместе с тем вдали и от внутреннего (вязкого) масштаба $l$, где энергия рассеивается. Важность этого вопроса обусловлена хорошо известным экспериментальным фактом, что процессы гомогенизации начальных и граничных условий (энергетической накачки на больших масштабах), приводящие к развитой турбулентности, являются значительно более медленными, чем считалось ранее (см., например, работу [10] и приведенную там литературу).

В связи с этим в настоящей работе мы исследуем процессы переноса слабого магнитного поля турбулентной средой, описываемой стохастическим уравнением Навье-Стокса, под воздействием спиральной случайной силы, т. е. случайной силы с нарушением пространственной четности, в рамках кинематической МГД-турбулентности. Наша цель состоит в том, чтобы найти явную зависимость турбулентного магнитного числа Прандтля от параметра, который контролирует наличие спиральности в турбулентной системе. Как будет показано, наличие спиральности может оказывать влияние на значение турбулентного магнитного числа Прандтля и, следовательно, на свойства диффузионных процессов векторной (магнитной) примеси для сред с полностью развитой турбулентностью.

\section{2. ТЕОРЕТИКО-ПОЛЕВАЯ ФОРМУЛИРОВКА МОДЕЛИ}

Перенос пассивного соленоидального магнитного поля $\mathbf{b} \equiv \mathbf{b}(x), x \equiv(t, \mathbf{x})$, в спиральной турбулентной среде в рамках кинематической МГД-турбулентности описывается системой стохастических уравнений

$$
\begin{aligned}
& \partial_{t} \mathbf{b}=\nu_{0} u_{0} \triangle \mathbf{b}-(\mathbf{v} \cdot \partial) \mathbf{b}+(\mathbf{b} \cdot \partial) \mathbf{v}+\mathbf{f}^{\mathbf{b}} \\
& \partial_{t} \mathbf{v}=\nu_{0} \triangle \mathbf{v}-(\mathbf{v} \cdot \partial) \mathbf{v}-\partial P+\mathbf{f}^{\mathbf{v}}
\end{aligned}
$$


где используются стандартные обозначения: $\partial_{t} \equiv \partial / \partial t, \partial_{i} \equiv \partial / \partial x_{i}, \triangle \equiv \partial^{2}$ - оператор Лапласа, $\nu_{0}$ - коэффициент вязкости, $u_{0}$ - обратное магнитное число Прандтля, $\mathbf{v} \equiv \mathbf{v}(x)$ - несжимаемое поле скоростей, $P \equiv P(x)$ - давление. Произведение $\nu_{0} u_{0}=c^{2} / 4 \pi \sigma$ представляет собой магнитную диффузию, где $c-$ скорость света, a $\sigma$ - проводимость. Вследствие несжимаемости поле скоростей $\mathbf{v} \equiv \mathbf{v}(x)$ является соленоидальным, и, таким образом, как $\mathbf{v}$, так и $\mathbf{b}$ являются бездивергентными векторными полями: $\partial \cdot \mathbf{v}=\partial \cdot \mathbf{b}=0$.

Пусть случайный шум $\mathbf{f}^{\mathbf{b}}=\mathbf{f}^{\mathbf{b}}(x)$ имеет гауссово распределение с коррелятором

$$
D_{i j}^{b}(x ; 0) \equiv\left\langle f_{i}^{\mathbf{b}}(x) f_{j}^{\mathbf{b}}(0)\right\rangle=\delta(t) C_{i j}\left(\frac{|\mathbf{x}|}{L}\right) .
$$

Он представляет собой источник флуктуаций магнитного поля. В равенстве $(2) L-$ интегральный масштаб, связанный с соответствующим перемешиванием, а $C_{i j}-$ функция, конечная в пределе $L \rightarrow \infty$, хотя в дальнейшем ее точный вид для нас неважен. Единственное условие, которому нужно удовлетворить, заключается в том, что $C_{i j}$ быстро убывает при $|\mathbf{x}| \gg L$. В то же время, если предположить, что функция $C_{i j}$ зависит также от направления вектора $\mathbf{x}$, а не только от его абсолютного значения $|\mathbf{x}|$, то ее можно использовать для описания крупномасштабной анизотропии в системе. С другой стороны, существенным является явный вид трансверсальной случайной силы на единицу массы $\mathbf{f}^{\mathbf{v}}$. Обычно предполагается, что она имеет гауссово распределение с нулевым средним и коррелятором

$$
D_{i j}^{v}\left(x ; x^{\prime}\right)=\left\langle f_{i}^{\mathbf{v}}(x) f_{j}^{\mathbf{v}}\left(x^{\prime}\right)\right\rangle=\delta\left(t-t^{\prime}\right) \int \frac{d^{d} \mathbf{k}}{(2 \pi)^{d}} R_{i j}(\mathbf{k}) d_{f}(k) e^{i \mathbf{k} \cdot\left(\mathbf{x}-\mathbf{x}^{\prime}\right)},
$$

где $d$ - размерность пространства $\mathbf{x}$, а $R_{i j}(\mathbf{k})$ - трансверсальный проектор. Он описывает геометрические свойства случайной силы и в простейшем изотропном случае определяется как $R_{i j}(\mathbf{k})=\delta_{i j}-k_{i} k_{j} / k^{2}$. Функция энергетической накачки $d_{f}(k)$ выбирается в виде, который позволяет реализовать реалистичное инфракрасное (ИК) введение энергии в систему (с помощью крупномасштабных вихрей), и в то же время важно, что в асимптотике больших $k$ функция $d_{f}(k)$ должна иметь степенной вид. Последнее условие необходимо для применения теоретико-полевых методов РГ. Обоим условиям удовлетворяет функция [5], [6]

$$
d_{f}(k)=D_{0} k^{4-d-2 \varepsilon}
$$

с положительной амплитудой $D_{0}>0$ и показателем $0<\varepsilon \leqslant 2$, наиболее реалистическое значение которого $\varepsilon=2$ (подробнее см., например, работы [5], [6]). В формуле (3) требуемая ИК-регуляризация дается ограничением интегрирования снизу, а именно $k \geqslant m$, где $m$ соответствует некоторому другому интегральному масштабу. Будем предполагать, что $L \gg 1 / m$. Далее для удобства вместо $D_{0}$ полезно ввести новую затравочную постоянную взаимодействия $g_{0}$, определив ее как

$$
D_{0} \equiv g_{0} \nu_{0}^{3}
$$

Кроме того, $g_{0}$ представляет собой формальный малый параметр обычной теории возмущений, связанный с характеристическим ультрафиолетовым (УФ) масштабом 
импульсов $\Lambda$ (или внутренней длиной $l \sim \Lambda^{-1}$ ) соотношением $g_{0} \simeq \Lambda^{2 \varepsilon}$. Галилеевская инвариантность стохастической модели (1)-(3) гарантируется временно́й декорреляцией случайных сил (2) и (3).

Переход к спиральной жидкости соответствует отказу от сохранения пространственной четности, что формально выражается в том, что корреляционная функция задается как смесь тензора и псевдотензора. В нашем изотропном случае это означает, что трансверсальный проектор $R_{i j}(\mathbf{k})$ в уравнении (3) можно разделить на две части:

$$
R_{i j}(\mathbf{k})=P_{i j}(\mathbf{k})+H_{i j}(\mathbf{k})=\delta_{i j}-\frac{k_{i} k_{j}}{k^{2}}+i \rho \varepsilon_{i j l} \frac{k_{l}}{k},
$$

где $P_{i j}(\mathbf{k})=\delta_{i j}-k_{i} k_{j} / k^{2}$ - неспиральный стандартный трансверсальный проектор, a $H_{i j}(\mathbf{k})=i \rho \varepsilon_{i j l} k_{l} / k$ выражает наличие спиральности в потоке. Здесь $\varepsilon_{i j l}-$ полностью антисимметричный тензор Леви-Чивиты ранга 3 , а вещественный параметр спиральности $\rho$ характеризует величину спиральности. Согласно требованию положительной определенности корреляционной функции абсолютное значение $\rho$ должно лежать в интервале $|\rho| \in[0,1]$. С физической точки зрения ненулевая спиральная часть выражает существование в системе ненулевых корреляций $\langle\mathbf{v} \cdot \operatorname{rot} \mathbf{v}\rangle$.

Используя хорошо известную теорему (см., например, работу [6] и приведенную там литературу), стохастическую задачу (1)-(3) можно переписать в рамках теоретико-полевой модели с двойным набором полей $\Phi=\left\{\mathbf{v}, \mathbf{b}, \mathbf{v}^{\prime}, \mathbf{b}^{\prime}\right\}$ с функционалом действия

$$
\begin{aligned}
S(\Phi)=\frac{1}{2} & \int d t_{1} d^{d} \mathbf{x}_{1} d t_{2} d^{d} \mathbf{x}_{2} \times \\
& \times\left[v_{i}^{\prime}\left(x_{1}\right) D_{i j}^{v}\left(x_{1} ; x_{2}\right) v_{j}^{\prime}\left(x_{2}\right)+b_{i}^{\prime}\left(x_{1}\right) D_{i j}^{b}\left(x_{1} ; x_{2}\right) b_{j}^{\prime}\left(x_{2}\right)\right]+ \\
& +\int d t d^{d} \mathbf{x}\left\{\mathbf{v}^{\prime}\left[-\partial_{t}+\nu_{0} \triangle-(\mathbf{v} \cdot \partial)\right] \mathbf{v}+\right. \\
& \left.+\mathbf{b}^{\prime}\left[-\partial_{t} \mathbf{b}+\nu_{0} u_{0} \triangle \mathbf{b}-(\mathbf{v} \cdot \partial) \mathbf{b}+(\mathbf{b} \cdot \partial) \mathbf{v}\right]\right\}
\end{aligned}
$$

где $x_{l}=\left(t_{l}, \mathbf{x}_{l}\right), l=1,2, \mathbf{v}^{\prime}(x)$ и $\mathbf{b}^{\prime}(x)$ - вспомогательные трансверсальные поля, имеющие те же тензорные свойства, что и поля $\mathbf{v}(x)$ и $\mathbf{b}(x)$, а $D_{i j}^{b}$ и $D_{i j}^{v}$ задаются формулами (2) и (3) и предполагается необходимое суммирование по немым индексам.

Модель (7) соответствует стандартной теории возмущений с фейнмановскими диаграммами со следующими ненулевыми затравочными пропагаторами (в частотно-импульсном представлении):

$$
\begin{aligned}
\left\langle v_{i} v_{j}^{\prime}\right\rangle_{0} & =\left\langle v_{i}^{\prime} v_{j}\right\rangle_{0}^{*}=\frac{P_{i j}(\mathbf{k})}{-i \omega+\nu_{0} k^{2}}, & \left\langle v_{i} v_{j}\right\rangle_{0} & =\frac{g_{0} \nu_{0}^{3} k^{4-d-2 \varepsilon} R_{i j}(\mathbf{k})}{\left|-i \omega+\nu_{0} k^{2}\right|^{2}}, \\
\left\langle b_{i}^{\prime} b_{j}\right\rangle_{0} & =\left\langle b_{i} b_{j}^{\prime}\right\rangle_{0}^{*}=\frac{P_{i j}(\mathbf{k})}{i \omega+\nu_{0} u_{0} k^{2}}, & \left\langle b_{i} b_{j}\right\rangle_{0} & =\frac{C_{i j}(\mathbf{k})}{\left|-i \omega+\nu_{0} u_{0} k^{2}\right|^{2}}
\end{aligned}
$$

где $C_{i j}(\mathbf{k})$ - фурье-образ функции $C_{i j}(\mathbf{r} / L)$ в соотношении $(2)$. С другой стороны, вершины взаимодействия в модели имеют вид $b_{i}^{\prime}\left(-v_{j} \partial_{j} b_{i}+b_{j} \partial_{j} v_{i}\right)=b_{i}^{\prime} v_{j} V_{i j l} b_{l}$ и $-v_{i}^{\prime} v_{j} \partial_{j} v_{i}=v_{i}^{\prime} v_{j} W_{i j l} v_{l} / 2$, где $V_{i j l}=i\left(k_{j} \delta_{i l}-k_{l} \delta_{i j}\right)$ и $W_{i j l}=i\left(k_{l} \delta_{i j}+k_{j} \delta_{i l}\right)$ (в импульсно-частотном представлении). Стандартное графическое представление пропагаторов и вершин можно найти, например, в работе [7]. 
В заключение данного раздела отметим, что преимущество формулировки стохастической задачи, задаваемой уравнениями (1)-(3) с функционалом действия (7), состоит в том, что она позволяет применять к анализу задачи корректно определенные теоретико-полевые методы, например методы РГ, и при этом статистические средние случайных величин в стохастической задаче заменяются соответствующими функциональными средними с весом $e^{S(\Phi)}[5],[6]$.

\section{3. РЕНОРМГРУППОВОЙ АНАЛИЗ}

РГ-анализ произвольной теоретико-полевой модели основан на анализе УФ-расходимостей, что, с другой стороны, определяется из анализа канонической размерности. Динамическая модель (7) принадлежит к классу так называемых двухмасштабных моделей [5], [6], т. е. к классу моделей, для которых каноническая размерность некоторой величины $Q$ задается двумя числами: импульсной размерностью $d_{Q}^{k}$ и частотной размерностью $d_{Q}^{\omega}$. Поэтому размерности всех величин можно найти, используя требование, чтобы каждый член функционала действия был безразмерным как по импульсу, так и по частоте, а также с учетом стандартных определений (условий нормировки) $d_{k}^{k}=-d_{x}^{k}=d_{\omega}^{\omega}=-d_{t}^{\omega}=1$ и $d_{k}^{\omega}=d_{\omega}^{k}=0$. Тогда полная каноническая размерность $d_{Q}$ определяется как $d_{Q}=d_{Q}^{k}+2 d_{Q}^{\omega}$ и играет ту же роль в теории перенормировки нашей динамической модели, что и простая импульсная размерность в статических моделях.

Размерный анализ действия (7) для пространственной размерности $d>2$ [8], [11] показывает, что модель является логарифмической при $\varepsilon=0$, т. е. постоянная взаимодействия $g_{0}$ в этой точке безразмерна, и что наивные УФ-расходимости имеют вид полюсов по $\varepsilon$ и представлены только в одночастично-неприводимых функциях $\left\langle v_{i}^{\prime} v_{j}\right\rangle_{1-\text { iг }}$ и $\left\langle b_{i}^{\prime} b_{j}\right\rangle_{1-\text { ir }}$ с контрчленами ${ }^{1)}: \mathbf{v}^{\prime} \Delta \mathbf{v}$ и $\mathbf{b}^{\prime} \Delta \mathbf{b}$. Их можно явно выразить в терминах мультипликативной перенормировки затравочных параметров $g_{0}, \nu_{0}$, и $u_{0}$ без перенормировки полей, а именно

$$
\nu_{0}=\nu Z_{\nu}, \quad g_{0}=g \mu^{2 \varepsilon} Z_{g}, \quad u_{0}=u Z_{u},
$$

где безразмерные параметры $g, \nu$ и $u$ представляют собой перенормированные контрпартнеры соответствующих затравочных параметров, $\mu$ - перенормировочная масса (параметр, задающий масштаб), являющаяся артефактом схемы регуляризации, а $Z_{i}=Z_{i}(g, u, \rho, d, \varepsilon), i=g, \nu, u,-$ так называемые константы перенормировки.

Перенормированный функционал действия имеет вид

$$
\begin{aligned}
S^{\mathrm{R}}(\Phi)= & \frac{1}{2} \int d t_{1} d^{d} \mathbf{x}_{1} d t_{2} d^{d} \mathbf{x}_{2} \times \\
& \times\left[v_{i}^{\prime}\left(x_{1}\right) D_{i j}^{v}\left(x_{1} ; x_{2}\right) v_{j}^{\prime}\left(x_{2}\right)+b_{i}^{\prime}\left(x_{1}\right) D_{i j}^{b}\left(x_{1} ; x_{2}\right) b_{j}^{\prime}\left(x_{2}\right)\right]+
\end{aligned}
$$

\footnotetext{
1) Важно подчеркнуть, что аналогично модели вещественной МГД-турбулентности (см., например, работу [12] и приведенную там литературу) в нашей модели со спиральностью могут появляться линейные расходимости. Правильное обращение с ними в истинной классической МГД-турбулентности приводит к появлению однородного крупномасштабного магнитного поля (турбулентный динамо-эффект), порожденного механизмом, похожим на механизм спонтанного нарушения симметрии. Однако этот механизм требует наличия члена с силой Лоренца в стохастическом уравнении Навье-Стокса. Поскольку в нашей модели такого члена нет, в настоящей работе мы не будем касаться проблемы линейных расходимостей. В то же время нам известно, что при наличии спиральности полную задачу можно решить только в рамках истинной классической МГД-турбулентности.
} 


$$
\begin{aligned}
& +\int d t d^{d} \mathbf{x}\left\{\mathbf{v}^{\prime}\left[-\partial_{t}+\nu Z_{1} \triangle-(\mathbf{v} \cdot \partial)\right] \mathbf{v}+\right. \\
& \left.+\mathbf{b}^{\prime}\left[-\partial_{t} \mathbf{b}+\nu u Z_{2} \triangle \mathbf{b}-(\mathbf{v} \cdot \partial) \mathbf{b}+(\mathbf{b} \cdot \partial) \mathbf{v}\right]\right\}
\end{aligned}
$$

где $Z_{1}$ и $Z_{2}$ - постоянные перенормировки, связанные с ранее определенными константами перенормировки в (9) следующим образом:

$$
Z_{\nu}=Z_{1}, \quad Z_{g}=Z_{1}^{-3}, \quad Z_{u}=Z_{2} Z_{1}^{-1},
$$

а $Z_{1}$ и $Z_{2}$ - независимые постоянные перенормировки (в используемой ниже схеме минимального вычитания), имеющие вид

$$
\begin{gathered}
Z_{1}(g, \rho, d, \varepsilon)=1+\sum_{n=1}^{\infty} g^{n} \sum_{j=1}^{n} \frac{z_{n j}^{(1)}(\rho, d)}{\varepsilon^{j}}, \\
Z_{2}(g, \rho, u, d, \varepsilon)=1+\sum_{n=1}^{\infty} g^{n} \sum_{j=1}^{n} \frac{z_{n j}^{(2)}(\rho, u, d)}{\varepsilon^{j}} .
\end{gathered}
$$

Здесь коэффициенты $z_{n j}^{(i)}, i=1,2$, не зависят от $\varepsilon$. Их явный вид можно определить, потребовав, чтобы одночастично-неприводимые функции Грина $\left\langle v_{i}^{\prime} v_{j}\right\rangle_{1-\mathrm{ir}}$ и $\left\langle b_{i}^{\prime} b_{j}\right\rangle_{1-\text { ir }}$ были конечными в УФ-пределе, если они записаны в перенормированных переменных, т. е. они должны быть свободны от полюсов по $\varepsilon$.

В случае отсутствия спиральности $(\rho=0)$ разложение постоянных перенормировки $Z_{i}, i=1,2$, известно с точностью до второго порядка по $g$ (двухпетлевое приближение) [8], [9], [11]. Простейший однопетлевой результат для $z_{11}^{(i)}, i=1,2$, имеет вид

$$
z_{11}^{(1)}=-\frac{S_{d}}{(2 \pi)^{d}} \frac{d-1}{8(d+2)}, \quad z_{11}^{(2)}=-\frac{S_{d}}{(2 \pi)^{d}} \frac{d-1}{4 d u(1+u)},
$$

где $S_{d}=2 \pi^{d / 2} / \Gamma(d / 2)$ - площадь поверхности $d$-мерной единичной сферы, а $\Gamma(x)-$ гамма-функция Эйлера. При отсутствии спиральности выражения для двухпетлевых поправок $z_{21}^{(i)}, i=1,2$, довольно громоздки, их можно найти в работах [8], [9], [11]. С другой стороны, связь коэффициентов $z_{22}^{(i)}, i=1,2$, с однопетлевыми коэффициентами $z_{11}^{(i)}, i=1,2$, простая, однако в дальнейшем они для нас неважны. Известна также явная зависимость коэффициента $z_{21}^{(1)}$ от параметра спиральности. Она была вычислена в работе [13]. Поэтому для анализа роли спиральности в рамках предлагаемой модели (в двухпетлевом приближении) необходимо найти явную зависимость коэффициента $z_{21}^{(2)}$ от параметра спиральности. Она получается путем явного вычисления соответствующих восьми двухпетлевых фейнмановских диаграмм (подробнее см. [7]). Окончательное численное выражение для коэффициента $z_{21}^{(2)}$ как функции параметра спиральности $\rho$ для пространства размерности $d=3$ (единственная пространственная размерность, соответствующая наличию спиральности) имеет вид

$$
z_{21}^{(2)}=-1.1375 \cdot 10^{-5}-0.3403 \cdot 10^{-6} \rho^{2},
$$

где уже использовано значение однопетлевой фиксированной точки для параметра $u_{*}^{(1)}=1.39297$ (см. ниже). 
Тот факт, что все поля модели не перенормируются, означает, что перенормированные корреляционные функции $W^{\mathrm{R}}=\langle\Phi \ldots \Phi\rangle^{\mathrm{R}}$ равны своим неперенормированным контрпартнерам $W=\langle\Phi \ldots \Phi\rangle$. Единственное различие заключается в выборе переменных (перенормированных или неперенормированных) и в соответствующем пертурбативном разложении (по $g$ или $g_{0}$ ). Используя тот факт, что неперенормированные корреляционные функции не зависят от задающего масштаб параметра $\mu$, немедленно получаем основное дифференциальное уравнение РГ

$$
\left[\mu \partial_{\mu}+\beta_{g}(g) \partial_{g}+\beta_{u}(g, u) \partial_{u}-\gamma_{\nu}(g) \nu \partial_{\nu}\right] W^{\mathrm{R}}(g, \nu, u, \mu, \ldots)=0,
$$

где так называемые функции РГ ( $\beta$ - и $\gamma$-функции) задаются как

$$
\begin{gathered}
\beta_{g} \equiv \mu \partial_{\mu} g=g\left(-2 \varepsilon+3 \gamma_{1}\right), \quad \beta_{u} \equiv \mu \partial_{\mu} u=u\left(\gamma_{2}-\gamma_{1}\right), \\
\gamma_{i} \equiv \mu \partial_{\mu} \ln Z_{i}, \quad i=1,2
\end{gathered}
$$

Напомним, что производная $\partial_{\mu}$ в уравнении (16) берется при фиксированных перенормированных параметрах, в то время как в соотноошениях (17) она берется при фиксированных затравочных параметрах.

ИК-асимптотическое скейлинговое поведение корреляционных функций модели (скейлинговое поведение глубоко внутри инерциального интервала) определяется ИК-устойчивой фиксированной точкой уравнений РГ. С другой стороны, фиксированная точка определяется из требования, чтобы в ней обращались в нуль $\beta$-функции $\beta_{g}$ и $\beta_{u}$ (в этом случае уравнения РГ (16)) становятся дифференциальными уравнениями для обобщенно-однородных функций $W^{\mathrm{R}}$, т. е. решения имеют скейлинговый вид). Таким образом, координаты фиксированной точки задаются системой уравнений

$$
\beta_{g}\left(g_{*}\right)=g_{*}\left(-2 \varepsilon+3 \gamma_{1}^{*}\right)=0, \quad \beta_{u}\left(g_{*}, u_{*}\right)=u_{*}\left(\gamma_{2}^{*}-\gamma_{1}^{*}\right)=0,
$$

где значения всех величин в фиксированной точке помечены звездочками. Это немедленно дает точные значения для аномальных размерностей $\gamma_{1}^{*}$ и $\gamma_{2}^{*}$ в фиксированной точке $g_{*} \neq 0, u_{*} \neq 0$, а именно $\gamma_{i}^{*}=2 \varepsilon / 3, i=1,2$, как результат того, что вид функций $\beta_{g}$ и $\beta_{u}$ в (17) не зависит от порядка разложения по теории возмущений, т. е. он уже точно определен в однопетлевом приближении в рамках теории возмущений без старших петлевых поправок.

Тем не менее, явный вид постоянных перенормировки $Z_{i}, i=1,2$, определяющих явный вид аномальных размерностей $\gamma_{i}(17)$, необходим для определения ИК-притяжения соответствующей фиксированной точки. Достаточным условием для ИК-устойчивости фиксированной точки является положительность вещественных частей собственных значений матрицы первых производных $\Omega_{k l}=\partial \beta_{k} / \partial l$, $k, l=g, u$, взятых в фиксированной точке $g_{*}, u_{*}$. Здесь необходим явный вид фиксированной точки. В случае наличия спиральности с точным значением $d=3$ координаты фиксированной точки суть

$$
g_{*}=\frac{40 \pi^{2}}{3} \varepsilon(1-1.0994 \varepsilon), \quad u_{*}=1.39297+\left(0.07260+0.03114 \rho^{2}\right) \varepsilon,
$$

где указана явная зависимость от параметра спиральности $\rho$. 
Прямыми вычислениями можно показать, что оба собственных значения матрицы первых производных имеют положительные вещественные части для всех разрешенных значений параметра спиральности $\rho$, а именно $|\rho|<1$. Таким образом, соответствующий ИК-скейлинговый режим является устойчивым.

\section{4. СПИРАЛЬНОСТЬ}

Теперь у нас имеются все результаты, необходимые для анализа влияния спиральности на турбулентное магнитное число Прандтля. В работе [7] было показано, что в двухпетлевом приближении РГ-формула для обратного турбулентного (эффективного) магнитного числа Прандтля $u_{\text {eff }}$ в модели кинематической МГД-турбулентности имеет тот же вид, что и соответствующее обратное турбулентное число Прандтля для пассивно увлекаемого скалярного поля [8], а именно

$$
\begin{aligned}
u_{\mathrm{eff}}=u_{*}^{(1)} & \left\{1+\varepsilon\left[\frac{1+u_{*}^{(1)}}{1+2 u_{*}^{(1)}}\left(\lambda-\frac{128(d+2)^{2}}{3(d-1)^{2}} B\left(u_{*}^{(1)}\right)\right)+\right.\right. \\
+ & \left.\left.\frac{(2 \pi)^{d}}{S_{d}} \frac{8(d+2)}{3(d-1)}\left(a_{v}-a_{b}\right)\right]\right\} .
\end{aligned}
$$

Здесь $u_{*}^{(1)}$ - однопетлевое значение параметра $u$ в фиксированной точке (равное однопетлевому значению обратного турбулентного магнитного числа Прандтля). В общем случае $d>2$ оно определяется простым уравнением

$$
u_{*}^{(1)}\left(1+u_{*}^{(1)}\right)=\frac{2(d+2)}{d},
$$

и при $d=3$ получаем $u_{*}^{(1)}=1.39297$. Значение $\lambda$ связано с коэффициентом $z_{21}^{(1)}$ в формуле (12), т. е. оно получается из двухпетлевого РГ-анализа модели чистой полностью развитой турбулентности, основанной на стохастическом уравнении Навье-Стокса, которое было получено в работе [11] без учета спиральности, а в работе [13] была проанализирована его зависимость от параметра спиральности. В работе [13] было показано, что несмотря на то, что все двухпетлевые диаграммы явно зависят от параметра спиральности $\rho$, окончательное выражение для $\lambda$ не зависит от $\rho$. Таким образом, при $d=3$ имеем $\lambda=-1.0994$ как в отсутствие, так и при наличии спиральности. Далее, величины $a_{v}, a_{b}$ даются соответствующими разложениями в главном порядке по $\varepsilon$ скейлинговых функций для соответствующих функций отклика $\left\langle v v^{\prime}\right\rangle$ и $\left\langle b b^{\prime}\right\rangle$ для поля скоростей и магнитного поля (подробнее см. [7], [8]). Их значения при $d=3$ (как при наличии, так и в отсутствии спиральности) равны

$$
a_{v}=-\frac{0.047718}{2 \pi^{2}}, \quad a_{b}=-\frac{0.04139}{2 \pi^{2}} .
$$

Наконец, величина $B\left(u_{*}^{(1)}\right)$ в формуле $(20)$ при $d=3$ (с учетом спиральности) простым образом связана с коэффициентом $z_{21}^{(2)}$, заданным в (15) (подробнее см. [7]):

$$
B\left(u_{*}^{(1)}, \rho\right)=\frac{(2 \pi)^{6}}{S_{3}^{2}} z_{21}^{(2)}=-4.4320 \cdot 10^{-3}-0.1326 \cdot 10^{-3} \rho^{2} .
$$


Таким образом, используя все определенные выше величины и полагая $d=3$, получаем следующее окончательное двухпетлевое выражение для обратного турбулентного магнитного числа Прандтля в турбулентной среде со спиральностью:

$$
u_{\mathrm{eff}}=1.39297+\left(0.013835+0.031143 \rho^{2}\right) \varepsilon+O\left(\varepsilon^{2}\right)
$$

а для физического значения $\varepsilon=2$ окончательно получаем двухпетлевое значение турбулентного магнитного числа Прандтля как функцию спиральности:

$$
\operatorname{Pr}_{\mathrm{m}, \mathrm{t}}=u_{\mathrm{eff}}^{-1}=\frac{1}{1.42064+0.06229 \rho^{2}}
$$

В пределе $\rho \rightarrow 0$ в среде без спиральности получаем значение ${ }^{2)} \operatorname{Pr}_{\mathrm{m}, \mathrm{t}}=0.7039$. С другой стороны, в полностью спиральном случае, т. е. при $|\rho|=1$, имеем $\operatorname{Pr}_{\mathrm{m}, \mathrm{t}}=$ 0.6743. Таким образом, можно видеть, что в турбулентной среде со спиральностью, т. е. при возрастании абсолютного значения параметра $\rho$, турбулентное магнитное число Прандтля убывает. В то же время при наличии спиральности двухпетлевые вклады в турбулентное магнитное число Прандтля достигают $4.2 \%$ (в случае максимальной спиральности) от их значения в отсутствии спиральности.

\section{5. ЗАКЛЮЧЕНИЕ}

В настоящей работе мы использовали теоретико-полевой подход РГ в двухпетлевом приближении для анализа влияния спиральности (нарушения пространственной четности) в потоках с полностью развитой турбулентностью на диффузионные процессы в пассивно увлекаемом магнитном поле в рамках кинематической МГД-турбулентности. Найдено турбулентное магнитное число Прандтля как функция параметра спиральности. Показано, что спиральность уменьшает значение турбулентного магнитного числа Прандтля. Хотя в спиральном случае двухпетлевые поправки к турбулентному магнитному числу Прандтля довольно малы (до $4.2 \%$ от их значения в отсутствии спиральности), оказалось, что отличия полученных результатов от результатов для полностью симметричных турбулентных систем могут оказывать нетривиальное влияние на универсальные характеристики процессов в турбулентных средах.

Благодарности. Авторы благодарят за гостеприимство Лабораторию теоретической физики им. Н. Н. Боголюбова Объединенного института ядерных исследований (Дубна, Россия). M. Юрчишин также благодарит за гостеприимство CERN, Theoretical Group of Physics Department. Работа поддержана Slovak Academy of Sciences (VEGA грант № 2/0093/13) и проектом ITMS № 26220120029, программа исследования и развития финансируется European Regional Development Fund.

2) Следует заметить, что это значение несколько отличается от значения 0.7051, полученного в работе [7]. Это связано с тем, что в настоящей работе мы использовали более точное, чем в работе [7], численное значение для величины $\lambda$, а именно $\lambda=-1.0994$. В работе [7] использовалось менее точное значение $\lambda=-1.101$, вычисленное в работе [11]. 


\section{Список литературы}

[1] A. Yoshizawa, S.-I. Itoh, K. Itoh, Plasma and Fluid Turbulence: Theory and Modelling, IoP, Bristol-Philadelphia, 2003.

[2] D. Biskamp, Magnetohydrodynamic Turbulence, Cambridge Univ. Press, Cambridge, 2003.

[3] A.S. Monin, A. M. Yaglom, Statistical Fluid Mechanics: Mechanics of Turbulence, Dover, Mineola, NY, 2007.

[4] L. P. Chua, R. A. Antonia, Internat. J. Heat Mass Transfer, 33 (1990), 331-339; K.-A. Chang, E. A. Cowen, J. Eng. Mech., 128:10 (2002), 1082-1087.

[5] L. Ts. Adzhemyan, N. V. Antonov, A. N. Vasil'ev, The Field Theoretic Renormalization Group in Fully Developed Turbulence, Gordon and Breach, London, 1999.

[6] А.Н. Васильев, Квантовополевая ренормгруппа в теории критического поведения и стохастической динамике, Изд-во ПИЯФ, СПб., 1998.

[7] E. Jurčišinová, M. Jurčišin, R. Remecký, Phys. Rev. E, 84:4 (2011), 046311, 11 pp.

[8] L. Ts. Adzhemyan, J. Honkonen, T. L. Kim, L. Sladkoff, Phys. Rev. E, 71:5 (2005), 056311, 9 pp., arXiv: physics/0412019.

[9] E. Jurčišinová, M. Jurčišin, R. Remecký, Phys. Rev. E, 82:2 (2010), 028301, 3 pp.

[10] G. Falkovich, K. Gawȩdzki, M. Vergassola, Rev. Modern Phys., 73:4 (2001), 931-975; L. Biferale, I. Procaccia, Phys. Rep., 414:2-3 (2005), 43-164; N. V. Antonov, J. Phys. A, 39:25 (2006), 7825-7865.

[11] L. Ts. Adzhemyan, N. V. Antonov, M. V. Kompaniets, A. N. Vasil'ev, Internat. J. Modern Phys. B, 17:10 (2003), 2137-2170.

[12] M. Hnatich, M. Jurcisin, M. Stehlik, Magnetohydrodynamics, 37:1-2 (2001), 80-86.

[13] E. Jurčišinová, M. Jurčišin, R. Remecký, Phys. Rev. E, 79:4 (2009), 046319, 10 pp.; 86:4 (2012), 049902, 1 pp. 